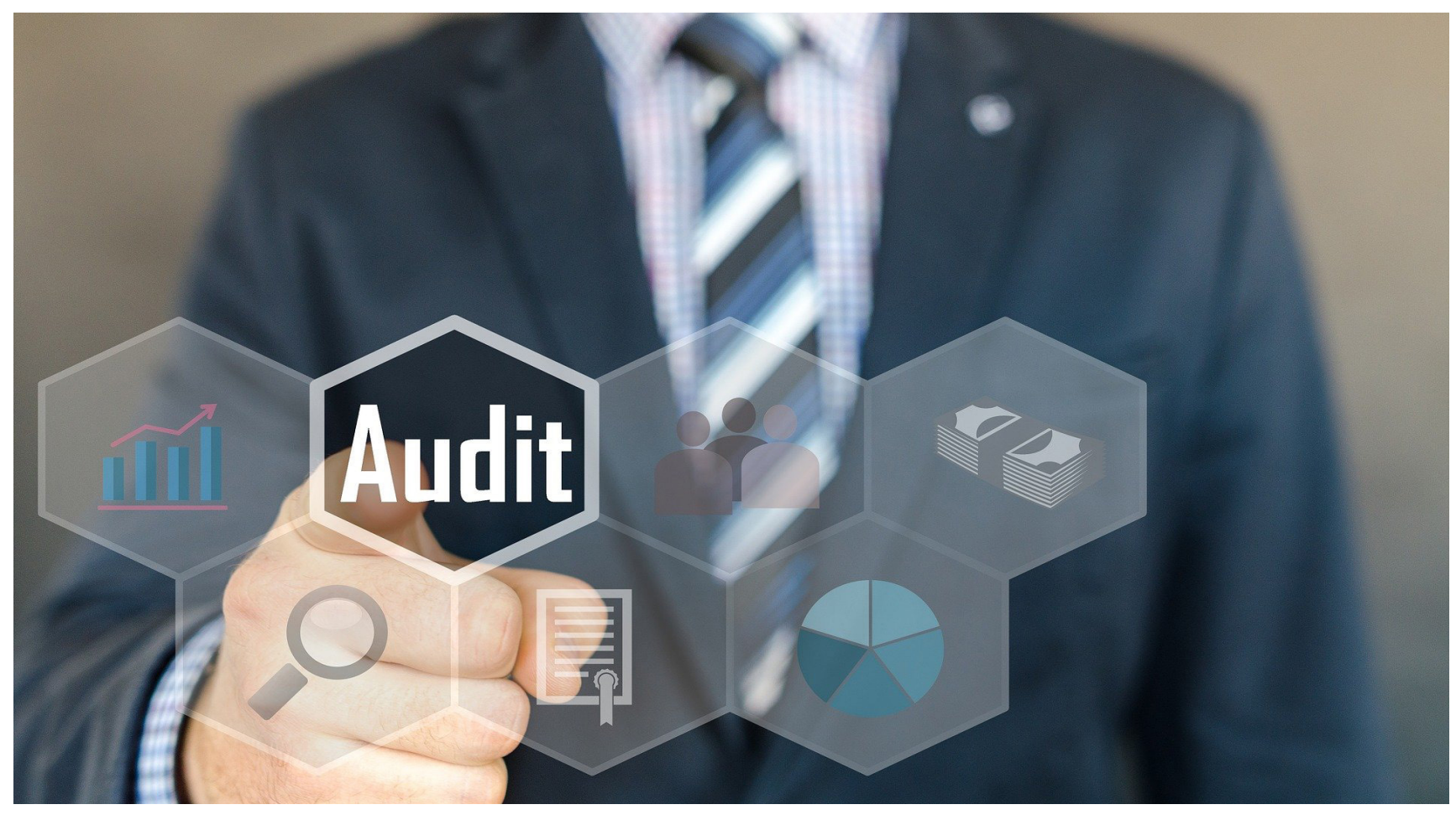

\title{
Influencia de la Auditoría forense en la detección del fraude en Colombia
}

\section{Influence of the forensic audit in the detection of fraud in Colombia}

Ruby Alejandra Acosta-Ramírez

Estudiante del Programa de Contaduría Pública, Universidad

Francisco de Paula Santander, Cúcuta, Colombia

Luis Fernando Guardiola-Plazas

Magister en Gerencia de Empresas mención Finanzas, luisfernandogp@ufps.edu.co, Universidad Francisco de Paula Santander, Cúcuta, Colombia 
Cómo citar: Acosta-Ramírez, R.A., Guardiola-Plazas, L.F. (2019). Influencia de la Auditoría forense en la detección del fraude en Colombia. Reflexiones contables (Cúcuta), 2 (2), 87-101.

\section{Resumen}

En Colombia se han conocido diversos casos de fraude y corrupción que afectan la prestación efectiva de algunos servicios o la desviación de los recursos destinados a infraestructura y otras áreas que impiden su crecimiento y desarrollo económico. Este artículo tiene como objetivo conocer la influencia de la Auditoría forense en la prevención del fraude en Colombia, por lo cual es indispensable hacer una introducción a la auditoría en general, de la cual se despliega la auditoría forense con una descripción del fraude que es uno de sus objetivos de esta auditoría, así mismo se presentan las actividades se han desplegado con relación a la auditoría por medio de las normas, así como la participación de entidades públicas y privadas en la orientación y diseño de procedimientos que permitan tener un marco de actuación para prevenir el fraude, y por último, mostrar ejemplos de casos presentados en Colombia donde la auditoría forense ha incidido para aplicar las medidas penales correspondientes a través de la recolección de pruebas, con el fin de mostrar la importancia de esta actividad. La información muestra que la auditoría forense ha sido de gran apoyo para el esclarecimiento de hechos de corrupción y fraude presentados en el país a partir de su labor investigativa y evidencias encontradas como soporte para la determinación judicial. En conclusión, si bien en los casos presentados ha habido resultados que ponen al descubierto las maniobras utilizadas por los responsables de estos actos indebidos, se hace difícil la compensación de los recursos; a pesar de que obtener el material probatorio suficiente es una de las funciones de la auditoría forense es importante apuntar a la etapa preventiva, de modo tal que se eviten estos acontecimientos.

Palabras clave: Auditoría, fraude, forense, corrupción, organización.

\section{Abstract}

In Colombia, various cases of fraud and corruption have been known that affect the effective provision of some services or the diversion of resources destined for infrastructure and other areas that impede their growth and economic development. This article aims to know the influence of forensic audit in the prevention of fraud in Colombia, for which it is essential to make an introduction to auditing in general, from which the forensic audit is displayed with a description of the fraud that is one of its objectives of this audit, as well as the activities that have been deployed in relation to the audit through the standards, as well as the participation of public and private entities in the orientation and design of procedures that allow having a framework of action to prevent fraud, and finally, to show examples of cases presented in Colombia where the forensic audit has influenced to apply the corresponding criminal measures through the collection of evidence, in order to show the importance of this activity. The information shows that the forensic audit has been of great support for the clarification of corruption and fraud facts presented in the country based on its investigative work and evidence found as support for the judicial determination. In conclusion, although in the cases presented there have been results that reveal the maneuvers used by those responsible for these improper acts, the compensation of resources is difficult; Although obtaining sufficient probative material is one of the functions of the forensic audit, it is important to aim for the preventive stage, so that these events are avoided. Key words: Audit, fraud, forensic, corruption, organization. 


\section{Introducción}

Hoy en día el mundo presenta ambientes altamente competitivos entre las organizaciones que buscan ocupar un lugar privilegiado en el mercado, lo cual las obliga a diseñar estrategias que permitan aprovechar al máximo las oportunidades que allí se presenten. Esta situación ayuda a impedir la generación de monopolios que impongan condiciones demasiado exigentes para sus consumidores (Monge, 2010).

Por otro lado, crea la necesidad de ejecutar sus actividades eficientemente para no desaprovechar o evitar que se desvíen los recursos invertidos, puesto que un capital mal ejecutado o desatendido puede marcar la diferencia en los bienes o servicios que la organización presta a sus clientes y que puede generar consecuencias dentro del desarrollo de los procesos o en la interacción de todas las áreas para la obtención de los logros propuestos (Moncayo C. , 2016).

Ante ello, fuentes internas y como externas generan sistemas de control que permitan minimizar los riesgos de ocurrencia de estos hechos, con el fin de maximizar sus beneficios y como medio estabilidad empresarial que marque el fortalecimiento de su estructura, reflejada en los resultados obtenidos, puesto que estos últimos son un atractivo para la inversión (criminalidad, 2011).

Si bien las empresas cuentan con un capital que les permite la realización de sus actividades para la generación de ingresos, siempre existe la necesidad de crecer, expandirse y tomar mayor posesión del mercado, por lo cual necesitan proveedores de financiamiento a través de la deuda o la generación de mecanismos para atraer la inversión. Sin embargo, antes de financiar un proyecto, los inversionistas estudian las posibilidades que existen en el mercado, basándose no sólo en la que pueda ofrecer una rentabilidad mayor, sino también en empresas que tengan una estabilidad constante que le permita minimizar los riesgos de fracasar en el proyecto y por ende afectar los intereses de los inversionistas (Santamaría, 2020).

\section{Orígenes de la auditoría}

Las actividades comerciales demandan muchas responsabilidades desde su proceso productivo hasta la generación de ingresos, donde cada elemento del talento humano es indispensable dentro de cada área de acuerdo al conocimiento y experiencia obtenida.

Sin embargo, estas funciones deben ser monitoreadas continuamente para evitar el riesgo al error (Valle, 2012).

En primera instancia dentro de la organización se diseñan controles para el cumplimiento efectivo de los roles asignados, sin embargo, al ser sus responsables, integrantes del ambiente interno, su labor puede estar siendo inducida por la gerencia.

Debido a ello, existen empresas que para verificar el funcionamiento interno, acuden a fuentes externas por cuanto su actividad tiene un mayor grado de imparcialidad con relación a la defensa de los intereses de los administradores (Rodríguez, 2016).

En esta situación juega un papel importante los controles que la organización diseñe para la optimización de los procesos y cuya responsabilidad recae en la gerencia y son evaluados a través de un diagnóstico por parte de especialistas como lo puede ser el profesional de Contaduría, con relación los hechos presentados. Es aquí donde se configura el trabajo de auditoría (Triana, 2014).

Desde el punto de vista del idioma inglés, auditar significa intervenir o revisar, pero de 
acuerdo al latín significa oír y tiene relación con que en primera instancia se juzgaba la verdad o la mentira a través de lo que los auditores escucharan.

Inicialmente, en la época de la revolución industrial, la vigilancia sólo estaba establecida para impedir que el personal incurriera en un error o pudiera cometer un fraude puesto que lo que existía en ese momento, eran pequeños negocios que no realizaban operaciones en cantidad.

Posteriormente éstos empiezan a crecer y la función se centra además de la anterior, en verificar la veracidad de la información que los responsables suministraban a través de los resultados. Seguidamente, la tecnología empieza a hacer parte del proceso, toda vez que las cantidades de transacciones se incrementan, allí la información contable debía garantizar el estado actual de la empresa y se empieza a evaluar el control interno. Hoy en día se extiende a la información que se presenta en los estados financieros, comprobando que esta sea transparente y razonable, donde se emite un informe que puede tener un concepto favorable o desfavorable (Izquierdo, 2016).

La auditoría puede entenderse como la acción de evaluar en qué medida las actividades desarrolladas por la empresa se ajustan a las políticas internas enmarcadas dentro de un marco legal.

Es un examen realizado por una persona interna de la organización o por un grupo de expertos en el área independientes de la empresa, sus resultados son transmitidos en una opinión (Lozano, 2014).

Es la revisión de la información generada por una empresa para establecer su veracidad como instrumento para obtener mayores beneficios. Es el examen que le permite al auditor establecer la autenticidad de los registros efectuados por la organización en sus actividades (Galvis, 2006).

La auditoría tiene por objeto a través del auditor, comprobar el cumplimiento de los lineamientos establecidos por la organización y la normatividad vigente de acuerdo a los soportes documentales para emitir una opinión o recomendación según sea el caso, la cual debe estar basada en los valores éticos que incluyen mantener una independencia con relación a su juicio profesional (Ochoa, 2016).

Esta necesidad surge porque la globalización amplió el margen de interesados de la información financiera que genera una empresa, obligando a garantizar la transparencia de los hechos reales con los que se presentan en los Estados Financieros, que al ser preparados bajo la supervisión de la gerencia pueden ser guiados al error o la intención de sacar provecho para defender sus intereses. En este sentido, de acuerdo a cada área de atención, se ha establecido un tipo de control (Curiel, 2006).

\section{La auditoría Forense}

Todas las actividades y de servicios que se realizan tienen como principal involucrado el factor dinero, como actor en la consecución de los medios necesarios para la supervivencia en el mundo. Este aspecto impulsa situaciones positivas como la creación de estrategias para la generación de ingresos, pero de igual forma medio distorsivos para obtenerlos como el fraude, ante el aprovechamiento del grado de confianza otorgado en las funciones asignadas por los dueños o accionistas de la empresa (Crespo, 2009).

Cuando se presentan desviaciones en la administración de una organización se ve afectada la sociedad en su conjunto, toda vez que en primera instancia quien sufre los efectos del fraude es la empresa en la pérdida 
de recursos que pueden disminuir su despliegue operativo y el Estado que lo componen todos, ya que estos recursos disminuyen directamente el aporte que hace la empresa por medio de los impuestos a través de la omisión de cifras e indirectamente ante el saqueo del capital de la empresa, en ambos casos reduciendo la contribución a pagar (Magro, 2004).

El fraude no guarda relación con el grado de progreso de los países, sino que abarca todos los niveles y se mueve y evoluciona de igual forma que lo hacen los procesos, procedimientos y la tecnología, a través de la creación de nuevos mecanismos para no ser detectados (Cano M. A., 2020).

Esta situación obliga tanto a las empresas como al gobierno a diseñar nuevas ideas que permitan descubrir este tipo de conductas de forma más rápida, no toma gran relevancia la aplicación de la auditoría forense, cuyo campo de acción está determinado en la parte financiera (Martinez, 2015).

Cada día estos comportamientos demandan mejoras en los procedimientos para su detección, que intenten reducir el margen de impunidad, toda vez que representan delitos como el fraude, el lavado de activos o la corrupción; además de las destrezas, conocimiento y experiencia en el área de quienes se encargan de la investigación (Luna, 2011).

El fraude puede establecerse en varias modalidades, como en la presentación de los estados financieros a través de la inexactitud provocada intencionalmente de la información ya sea por la omisión o la alteración de los datos, generalmente para la reducción de impuestos; en la alteración de activos que tienen como objetivo el robo de los mismos, donde está inmerso el personal interno responsable de sus administración, sin embargo, se extiende a condiciones externas con la participación de clientes y proveedores con apoyo del personal interno y; en el fraude orgánico que pretende atentar contra el negocio directamente (Suárez, 2019).

La auditoría forense se especializa en encontrar los medios que pueden constituirse en prueba como soporte ante un proceso legal. El auditor externo es solicitado en algunas empresas para evaluar el grado de efectividad que cumplen los protocolos diseñados para evitar que se cometa el fraude.

Esta auditoría es una práctica que investiga la ocurrencia de actos contrarios a la ley, mediante la utilización de herramientas de la criminalística y que además integran especialidades como el derecho, la contabilidad dentro de las cuales el personal cuenta con la suficiente idoneidad, con el fin de aportar información a la administración de justicia (Saldarriaga, 2011).

La auditoría forense es considerada una herramienta útil en el desarrollo de las actividades propias de abogados, unidades de policía, fiscalía y de la rama judicial, dando una visión clara ante los actos contrarios a la normatividad. Al servir de apoyo en diferentes ámbitos, de igual forma está integrada por un equipo especializado en diferentes áreas como la informática, el derecho y la grafología.

La auditoría forense se puede entender como la ciencia que se encarga de recolectar la información necesaria en los ámbitos contable, financiero, administrativo y legal para ser aportada como prueba ante la posible comisión de una conducta punible (Carlos Ocampo, 2010).

Es utilizada para identificar o prevenir acciones que conduzcan a la corrupción o al fraude y si es el caso presentar la información correspondiente ante los tribunales (Florez, 2009). 
Es un proceso estructurado guiado por el profesional en auditoría forense que requiere de la participación activa de su grupo de acuerdo a la especialidad para obtener un resultado más efectivo y contundente que permita demostrar los hechos fraudulentos presentados.

El profesional de auditoría es multidisciplinario y puede aportar en la claridad de asuntos empresariales como el fraude, lavado de activos, diferencias entre los socios, así como en casos relacionados con seguros o circunstancias familiares.

La auditoría forense tiene como objetivos identificar la consumación del hecho contrario a la ley, así como de prevenirlo o reducirlo a través de la orientación brindada a los responsables del control interno, promover estrategias preventivas, evaluar el sistema de control interno, recolectar pruebas a través de la investigación, así como servir de soporte técnico a los órganos fiscales y judiciales (Vaca, 2020).

De acuerdo a lo descrito anteriormente, la auditoría forense puede dividirse de acuerdo a dos objetivos, uno en la prevención y el otro de detección. En el primero, se enfoca en brindar asesoría a las empresas a través de la implementación de estrategias disuasivas y de control para evitar que se cometan los frauden o cualquier otro tipo de delito que atente contra la estabilidad y funcionamiento de la organización intervenida. En el segundo, los hechos ya están cometidos y la función es recolectar el máximo de información que implique la asignación de los responsables a través de los elementos que se puedan convertir en una prueba fidedigna $y$ contundente, sin embargo en muchos casos los datos recolectados no tienen un carácter definitivo, sino que quedan a disposición de los órganos judiciales para su evaluación y decisión (Piracón, 2020).
El auditor para cumplir con los objetivos de la auditoría forense debe analizar la información minuciosamente, ser creativo, contar con los conocimientos tanto en el área de los negocios como en las herramientas ofimáticas, ser discreto y que brinde la mayor confianza (Suárez, 2019).

El término auditoría hace referencia a la verificación de la información contable de una organización con el fin de establecer la autenticidad de las partidas con el fin de emitir una opinión en la gestión de la administración; por otro lado, el término forense significa plaza pública o foro y hace referencia a los juicios que se hacía en la plaza pública para la administración de justicia contra las personas con ocasión a los negocios que se realizaban (Cano, 2003).

La auditoría forense puede entenderse como el asesoramiento de expertos a los encargados de dictar sentencia ante la investigación de hechos catalogados como delitos ante la ley y que tienen relación con la parte financiera de una organización; de igual forma evalúa las conductas que no son normales a partir de las múltiples técnicas de hallazgo para obtener pruebas legales (Ramírez, 2013).

La gerencia determina el campo de acción de la auditoría forense ya sea de forma preventiva en la instrucción o asesoramiento o en la correctiva al encontrar evidencias y servir de apoyo durante un proceso judicial (Juan Caballero, 2018).

Un aspecto importante que tiene una labor antecesora a la auditoría forense es el control interno de las empresas, cuya función consiste en mitigar la posible comisión de hechos fraudulentos por parte del personal interno o como intermediarios ante terceros. Este proceso es de constante retroalimentación, que ayuda a corregir las falencias que el mismo 
sistema va encontrando en el desarrollo normal de las actividades operativas (Mantilla, 2013).

Los resultados obtenidos por la auditoría o contabilidad forense hacen parte de los debates judiciales en los que se determina la culpabilidad de los involucrados en el proceso, por lo cual se puede decir que tiene un carácter penal. Inicialmente estaba enfocada al sector público pero de acuerdo a los avances por el tema de la globalización en el sector privado su utilización se ha hecho obligatoria(Arcila, 2019)

\section{¿La auditoría forense es un mecanismo que da respuesta a los hechos del fraude en Colombia?}

En Colombia se han presentado varios casos de corrupción, en los cuales, con la participación de la auditoría forense, han podido ser resueltos, algunos ejemplos son: DMG, Interbolsa, el carrusel de la contratación en Bogotá, devoluciones, favorecimientos Dian y Odebrecht (Guzmán, 2019).

\section{Caso DMG}

Esta organización empezó a realizar actividades en Colombia en el año 2005, donde los clientes tenían la posibilidad de multiplicar su dinero en un tiempo corto. Para las autoridades colombianas el uso de tarjetas prepago, empresas involucradas y el posicionamiento de marca significaba un hecho de fraude a la ley. En 2008 fue capturado su creador, año para el cual ya había captado alrededor de dos mil millones de dólares. Después de una larga audiencia con intervención del ministerio público y la fiscalía, fue declarado culpable por los delitos de lavado de dinero a través de su captación ilegal, por lo que fue recluido en una cárcel en Colombia para ser extraditado a los Estados Unidos para cumplir una condena de 9 años (Dinero, 2019).

\section{Caso Interbolsa}

Esta corredora de bolsa Colombiana que inicio con el cobro de comisiones al atraer clientes a la inversión. Posteriormente en alianza con otros intervinientes crearon la modalidad de aprovechar la baja cotización para invertir y el alta para vender. El fraude presentado partió del manejo de operaciones repos que consistían en la venta de un bien a un inversor con la condición de volverla a comprar en una fecha determinada que sin caer inicialmente en el fraude, aprovechándose de algunos vacíos de la norma, lo que generaba la inflación de sus valores, por lo que las acciones de la compañía se duplicaron.

Esta situación y las relaciones con otros inversionistas sin temor al establecimiento del riesgo le generó una iliquidez, a raíz de lo cual no pudo cumplir las obligaciones con los inversionistas, momento en el cual tuvo que ser intervenida por la superintendencia financiera (Cordero, 2018).

\section{Caso Carrusel de la Contratación}

Este grupo basaba su accionar en el saqueo de recursos a la ciudad de Bogotá en la contratación en obras públicas, logrando desviar grandes sumas de dinero sin dejar huellas (transmilenio, ambulancias y malla vial). Las investigaciones revelan que una parte de los recursos fueron enviados a paraísos fiscales y otros invertidos en Estados Unidos. Las autoridades lograron comprobar la participación de integrantes del consejo, a los que se les precisó condena, al igual que algunos contratistas. Estas actuaciones se pusieron en evidencia con el retraso en un sector de la obra de transmilenio, lo que terminó dando apertura de otras irregularidades que dejaron como resultado la captura de alrededor de 40 involucrados entre concejales, contratistas y congresistas aunque a algunos se les dictó prisión domiciliaria. Además de estos hechos 


\section{4}

están involucrados otros de ámbito nacional, sin embargo no se han obtenido las pruebas suficientes para esclarecerlos (Semana, 2020).

\section{Casos DIAN}

En primera instancia se trata de un fraude en la administración de impuestos que involucraba a funcionarios que habían ejercido labores allí y consistía en la devolución irregular del Impuesto al valor agregado que fue detectado al incrementarse progresivamente las devoluciones $y$ a un porcentaje desproporcionado. Anteriormente se habían abierto investigaciones, las cuales fueron cerradas por un soborno.

Del hecho resultaron privadas de la libertad 12 personas por cohecho, enriquecimiento y lavado de activos, cuya apropiación estaba por alrededor de 1 billón de pesos (Portafolio, 2011).

Por otro lado, se produjo la captura de funcionarios de la Entidad, quienes aceptaban dineros para favorecer a los contribuyentes a través de la disminución o de la eliminación de las responsabilidades tributarias en el pago de impuestos o de las sanciones (un 10\% de los valores que en realidad debían pagar), situación que fue puesta en evidencia por la denuncia de un contribuyente que sirvió para hacerle seguimiento a los involucrados a través de la Dirección de Investigación Criminal de la Policía Nacional y recaudar las pruebas necesarias (alrededor de 70.000 procesos de cobro por $\$ 4$ billones) para la imputación de cargos dentro de los que están concierto para delinquir, cohecho propio y por dar $u$ ofrecer y concusión. A las empresas que participaron se les abrió un expediente para extinción de dominio (Forense, 2018).

\section{Caso Odebrecht}

Compañía del sector de la construcción que extendió sus actividades a nivel mundial, que fue obteniendo reconocimiento por la construcción de plantas Hidroeléctricas y que su accionar llegó hasta Colombia en 1992. Sin embargo en el año 2016 salieron a luz pública denuncias por irregularidades en las contrataciones, que indicaban la utilización de sobornos en cada uno de los países que desarrolló actividades, para poder acceder a ellos. Situación que fue soportada con los registros que uno de sus empleados guardó al ser despedido y que entregó a los órganos de control de la justicia brasileña. De igual forma testimonio de uno de sus integrantes señala la participación de la fiscalía general de la nación en irregularidades presentadas en contrato con el grupo AVAL. En Colombia los sobornos fueron utilizados para financiar campañas políticas a nivel de senado y presidencia (Semana, 2017).

Además de los mencionados, otros casos corresponden a Reficar S.A (peculado por alrededor de $\$ 610.000$ millones al realizar un cambio en las condiciones contractuales que hizo que los riesgos del mismo recayera en la refinería, que estaban a cargo del contratista de la modernización de la obra); el programa de alimentación escolar pública para los niños más pobres (10 casos de apropiación de estos recursos por contratistas en el país, dentro de los cuales se han hallado responsables más de 50 personas por los delitos de peculado, contratación ilegal, y concierto para delinquir); los recobros al Fondo de solidaridad y garantías (cobros por parte de miembros de la entidad y del ministerio de protección a través de fachadas); el cartel de la chatarrización (involucra miembros de la Policía, Ministerio de Transporte y camioneros al realizar esta actividad de forma fraudulenta en la falsificación de documentos y creación de incidentes ficticios que dieran origen a la 
chatarrización y así cobrar comisiones, por un lado con vehículos tipo camión y un segundo caso vehículos de carga); Ecopetrol (convenios que se encuentran en investigación realizados con la Empresa Red País Rural de inversión social para los cuales no se realizaron estudios, que no fueron ejecutados pero que si fueron pagados); Panamá Papers (en Colombia 14 empresas con la participación de sus directivos y contadores por intermedio de una firma de abogados trasladaron dineros de forma ilegal hacia panamá alterando la contabilidad de la empresa, la investigación revela movimientos por cerca de 11 millones de dólares) y; el caso Saludcoop (utilización de los excedentes de salud proveniente de los beneficios no utilizados por los usuarios en el plan obligatorio de salud (POS), y posterior figura del no POS que cobraban al FOSYGA, sin embargo como estos servicios eran prestados por EPS, para hacer efectivo el cobro debían estar al día con estas entidades, pero como el flujo de efectivo era grande no pudieron responder y fueron intervenidos por la superintendencia de salud y otras entidades por el manejo indebido de los recursos ) (Forense R. H., 2018)

El gobierno, las instituciones y demás integrantes en la detección del fraude

Inicialmente, a nivel internacional mediante la declaración Estándar de auditoría N 53 de 1998 se establece la responsabilidad de los auditores en la búsqueda de hechos irregulares dentro de su actividad y en el cual se enmarcan los riesgos a tener en cuenta cuando se evalúa la comisión de un posible fraude.

En la actualidad la Norma Internacional de Auditoría $N^{\circ} 240$ orienta el protocolo de actuación de los auditores cuando en la valoración de los Estados Financieros de una organización está la posibilidad de establecer el fraude (Rodriguez, 2017).
La ley 43 de 1990 señala las competencias que tiene el contador público y que se encuentran las relacionadas con el área contable, incluyendo los servicios de auditoría, dentro de los cuales no deben tener una incidencia en la contratación de personal, es decir, que este proceso sea transparente para evitar favorecimientos que se puedan convertir en un riesgo para la organización. De igual forma hace referencia a las normas de auditoría en el que se hace énfasis en el juicio profesional de su examen para la rendición del informe, basado en la documentación recolectada (República, 1990).

Con la ley 1349 de 2009, se adoptan unos instrumentos en materia de contabilidad e información financiera, así como los medios de control que permitan su aseguramiento, el cual está relacionado con las funciones y procedimientos de auditoría y en cabeza del presidente de la república y con el apoyo de las instituciones en materia son los encargados de la expedición y orientación de las normas, donde se delegan funciones de supervisión y control para su cumplimiento (Senado, 2020).

A través del decreto 2420 de 2015 se expide el reglamento único que además de las normas contables, incluye el aseguramiento de la información, allí se integran las normas internacionales de auditoría, de control de calidad, de trabajos de revisión y de atestiguar, de servicios relacionados y de ética. Dentro de las actuaciones, los procedimientos se deben realizar bajo el marco regulatorio previsto en ese decreto del que hacen parte las NICC y las normas descritas anteriormente (Pública, 2015).

La universidad de San Buenaventura de la Ciudad de Bogotá en la orientación de sus estudiantes a través del seguimiento de los docentes, incentiva la generación de propuestas para el mejoramiento de los procesos en diversas áreas, en este sentido, 


\section{6}

en su documento (Mora, 2006) propone un manual de Auditoría Forense para las entidades bancarias en Colombia, basándose en los medios para la administración del riesgo y los conceptos de fraude y control, donde establece a partir de las funciones del personal profesional del área, un protocolo de actuación dividido en etapas: La planeación relacionada con la noción del funcionamiento de la empresa y su ambiente y la revisión del control interno; la ejecución en la recolección de pruebas de la comisión de un fraude que deben evaluarse para establecer su certeza como elemento probatorio $\mathrm{y}_{i}$ por último la rendición del informe en el cual se detallan los hallazgos para ser comunicados a los interesados, convirtiéndose en una herramienta importante para el sector.

Dentro del decreto 2555 de 2010 se dictan normas relacionadas con el sector financiero, especialmente de las funciones de la Superintendencia financiera, donde su oficina asesora de planeación tiene la responsabilidad del diseño del plan anticorrupción de la entidad, además de las acciones pertinentes para su implementación en colaboración con las demás áreas, de igual forma se establecen las funciones planeación, organización y verificación de la oficina de control interno (Pública G. N., 2010).

El gobierno nacional a través de la asignación de responsabilidades por medio del decreto 1474 de 2011 busca fortalecer las herramientas que permitan prevenir 0 investigar y sancionar hechos de corrupción en la gestión pública, a través de normas orientadoras para su control, las cuales incluyen medidas administrativas, penales y disciplinarias, así como las entidades reguladoras y de control, sin embargo, estas actividades también se extienden la parte privada (República P. d., 2020).
El Programa Presidencial de Modernización, Eficiencia, Transparencia y Lucha contra la Corrupción fue sustituido por el Departamento Administrativo de la Presidencia de la República la Secretaría de Transparencia por medio del decreto 4637 de 2011, el cual dentro de sus funciones tiene la tarea de establecer una metodología en la lucha contra la corrupción como guía para las entidades del sector público. En cumplimiento a lo dispuesto elaboró el documento "Estrategias para la Construcción del Plan Anticorrupción y de Atención al Ciudadano", al cual le debe hacer estricto seguimiento. Estas disposiciones fueron reglamentadas mediante el decreto 2641 de 2012 (Juriscol, 2012).

En la ley 1778 de 2016 se establecen las responsabilidades administrativas de las personas jurídicas con relación a hechos de corrupción transnacional, además de dictar otras disposiciones para combatir la corrupción, donde se incluyen acciones como el soborno de servidores públicos en el extranjero, su proceso de investigación y posterior régimen sancionatorio. Las hechos enmarcan los ejecutados por cualquiera de los miembros que hacen parte de la organización como de quienes actúen en su representación, para el caso de establecer las responsabilidades (OCCP, 2016).

La Facultad de estudios a distancia de la Universidad de Nueva Granada de Colombia, publicó un documento de orientación concerniente a la normatividad y aspectos relevantes de la auditoría forense, en el cual se establece el marco legal para el desarrollo de esta actividad por parte de los profesionales de auditoría y centrándose en aspectos como el crimen organizado, el crimen de cuello blanco, así como los procedimientos a seguir en la elaboración de la auditoría, además de la importancia del material probatorio recolectado en dicho proceso mediante el 
aseguramiento de la cadena de custodia (UMNG, 2020).

En una entrevista, la auditora forense (Guzmán, 2019) señala la necesidad de establecer un área de auditoría forense en las empresas para que los controles diseñados para la detección de las irregularidades financieras, sean más efectivos y por ende confiables. Así mismo señala que hoy en día la tecnología se presenta como un medio tanto para la comisión de estas conductas pero a la vez para realizar la oportuna intervención a través de la capacitación de sus responsables que eviten la realización de fraudes corporativos. Por otro lado señala la importancia que ha adquirido esta actividad tanto en la parte pública como privada, lo que la ha hecho consolidarse en Colombia, en la necesidad de establecer protocolos que den seguridad de las operaciones contables.

Por medio del documento "Política para la prevención del Fraude y la Corrupción" el Grupo (Sura, 2018) establece una metodología en la que a partir de la asignación de los responsables, se establece el desarrollo de actividades para prevenir el fraude, su evaluación de riesgo, así como su oportuna detección a través de las investigaciones y las formas en que se debe dar respuesta a este o a un hecho de corrupción, a través de la evaluación de los sistemas de control interno.

(Bancoldex, 2019) A través del Sistema de Gestión de Calidad promueve al interior de la entidad la prevención ante los riesgos de fraude y corrupción por medio de un programa que permite el análisis de estos componentes en su prevención y control o detección para la elaboración de un mapa de riesgos que permita combatir estos flagelos en la organización, además de elementos como el sistema de control interno, de atención al consumidor, el código de buen gobierno y de ética.
Consultores y asesores en procesos de auditoría como (Bonilla, 2020) con su experiencia nacional e internacional, sugiere un proceso enmarcado en 10 aspectos para prevenir el fraude y la corrupción: política de estado a todos los niveles, aplicación sin atenuantes de las normas y disposiciones reglamentarias, observancia permanente de la misión de la organización de todos sus integrantes, proceso de selección meritoria del personal para los cargos de acuerdo a los perfiles, estandarizar los procesos y procedimientos internos, utilización de las tecnologías de la información, buzón de sugerencias, quejas o reclamos para ser evaluados por los clientes, administración de los riesgos, interventoría a través de profesionales altamente capacitados y una política que respalde la auditoria interna.

El consejo Técnico de Contaduría pública a través de su artículo, hace énfasis en las responsabilidades del auditor en la prevención del fraude, donde además del cumplimiento de los objetivos en materia, se establece las fases para la evaluación del riesgo de fraude, la planificación en el sentido de observar los medios que conduzcan a ello y por otro lado el diseño de estrategias para mitigarlos, así mismo en la ejecución durante el proceso de auditoría y posterior a él (Moncayo, 2019).

\section{Conclusiones}

La auditoría abarca muchas áreas a través del despliegue operacional que permite el desarrollo la auditoría forense como un mecanismo de control en tres instancias importantes y progresivas: Prevención, Investigación y sanción.

La auditoría forense surge como una alternativa que permita dar respuesta oportuna a los riesgos que pueden influir en el desarrollo de las actividades normales de la empresa, donde el fraude representa una gran incidencia por los medios fáciles que ofrece de 


\section{8}

obtener una utilidad sin prever las circunstancias legales que acarrea.

La auditoría forense es una herramienta que garantiza si bien no la totalidad de las irregularidades, si un alto índice de prevención de que ocurran, por lo que se ha determinado la necesidad de ofrecer un espacio dentro de las áreas de la organización para la realización de estas funciones de forma permanente y no para hallar responsables cuando los hechos ya están consumados.

Muchas normas e iniciativas señalan la importancia de esta labor y diseñan un plan de acción, sin embargo, las modalidades de fraude están a la vanguardia de la tecnología y cada vez se modernizan para lograr su cometido.

En este sentido, cada empresa en particular debe tomar estas guías y disposiciones normativas legales, para de acuerdo con las actividades que desarrolle, diseñar un sistema de control con la asesoría de expertos en esta área si los recursos no alcanzan para contratarlos plenamente, además realizarse una evaluación constante a estos controles que cada día van a demandar cambios para dar una respuesta más efectiva a las condiciones que genera el ambiente.

Si bien en los casos presentados se han presentado resultados que dan con las medidas carcelarias a los responsables y otros tipos de sanción, en muchos de ellos los recursos no pueden ser compensados; además, a pesar que dentro de las funciones de la auditoría forense está la de investigar para obtener el material probatorio suficiente, lo ideal sería no llegar a esas instancias, sino que todas aquellas intenciones quedaran en la etapa preventiva, aunque de cierta forma, en este caso no se observaría lo relevante de la función de la auditoría puesto que es incierto establecer si se pretendían realizar acciones y que consecuencias generaría.
Lo importante es aprovechar al máximo las herramientas que se brindan, puesto que estas acciones no sólo perjudican a la empresa y sus socios y empleados, sino además al estado, ya sea porque los casos se presenten en entidades públicas como la mayoría de los ejemplos o en las privadas que disminuyen la contribución que ellas deben realizar a través del pago de los impuestos, o como en otros con la eliminación de estos aportes.

\section{Referencias}

Arcila, R. H. (2019). Auditoría forense (1 ed.). México: Ecoe Ediciones. Recuperado el 11 de noviembre de 2020, de http://www.ebooks7-24. com.bdbiblioteca.ufps.edu.co:2048/?il=9193

Bancoldex.(2019). Bancoldex. Recuperado el 13 de noviembrede2020, dehttps:/www.google.com/ arch?gs_8j0j7\&sourceid=chrome\&ie=UTF-8

Bonilla, M. (2020). Auditool. Recuperado el 13 de noviembre de 2020, de https://www.auditool. org/blog/sector-gobierno/2025-10-tips-depoliticas-para-prevenir-la-corrupcion-y-elfraude

Cano, M. A. (2020). Interamerican Community Affairs. Recuperado el 13 de noviembre de 2020, de http://interamerican-usa.com/ articulos/Auditoria/Fraud-Estaf-Neg.htm

Cano, R. C. (2003). Legis Xperta. Recuperado el 12 de noviembre de 2020, de https://xperta. legis.co/visor/temp_rcontador_6b65f0a5978c-48c1-893a-010be5flef47

Carlos Ocampo, O. T. (2010). Las Técnicas forenses y la Auditoría. Revista Ciencia y Técnica. Recuperado el 12 de noviembre de 2020, de https://www.redalyc.org/ pdf/849/84917249019.pdf

Cordero, Y. D. (2018). Fundación Universitaria Empresarial de la Cámara de Comercio de 
Bogotá. Recuperado el 13 de noviembre de 2020, de https://bibliotecadigital.ccb.org.co/ bitstream/handle/11520/21081/TFCE\%20 S964i.pdf?sequence

Crespo, J. A. (2009). Detección del fraude en una auditoríoa de Estados Financieros. Revista Perspectivas, 227-242. Recuperado el 12 de noviembre de 2020, de https://www.redalyc. org/pdf/4259/425942160012.pdf

criminalidad, C. I. (2011). Oficina de las Naciones Unidas contra la droga y el delito. Recuperado el 14 de noviembre de 2020, de https://www. unodc.org/documents/justice-and-prisonreform/crimeprevention/Handbook_on_the Crime_Prevention_Guidelines_Spanish.pdf

Curiel, G. S. (2006). Auditoría de Estados Financieros (2 ed.). Mexico: Pearson Prentice Hall. Recuperado el 12 de noviembre de 2020, de https://books.google.es/s\&lr=\&id=xga7Z $6 z v j N Y C \& o i=$ fnd\&pg $=$ PA1\&dq $=$ auditoria $\&$ ots $=$ OscZm4Ta7p\&sig=KE9ouvWR0k6Nq bvJr4G6 $\operatorname{cinHGA} \# \mathrm{v}=$ onepage $\& \mathrm{q} \& \mathrm{f}=$ false

Dinero. (25 de junio de 2019). Dinero. Recuperado el 13 de noviembre de 2020, de https://www.dinero. $\mathrm{com} /$ pais/articulo/david-murcia-el-creador-dedmg-es-deportado-a-colombia/270167

Florez, A. E. (2009). Auditoría Forense. Revista de la Facultad de Ciencias Contables, 67-93. Recuperado el 13 de noviembre de 2020, de https://revistasinvestigacion.unmsm.edu.pe/ index.php/quipu/article/download/4825/3890/

Forense, H. (28 de septiembre de 2018). Huella Forense. Recuperado el 14 de noviembre de 2020, de https://www.huellaforense.com/ noticias/capturan-funcionarios-de-la-dian-porpresunto-cobro-de-sobornos-a-contribuyentes

Forense, R. H. (14 de diciembre de 2018). Huella
Forense. Recuperado el 14 de noviembre de 2020, de https://huellaforense.com/noticias/12casos-de-corrupcion-que-acosan-a-colombia

Galvis, O. d. (2006). El deber ser de la Auditoría. Recuperado el 11 de noviembre de 2020, de http://www.scielo.org.co/scielo.php?script=sci arttext\&pid=S0123-59232006000100004

Guzmán, L. (28 de marzo de 2019). Actualicese. Recuperado el 13 de noviembre de 2020, de https://actualicese.com/contaduria-debeenriquecerse-del-modus-operandi-de-redesde-lavado-de-activos-y-fraude-corporativo/

Izquierdo, K. L. (02 de noviembre de 2016). La auditoría forense: origen y aproximación como ciencia. Revista Universidad Externado de Colombia. Recuperado el 12 de noviembre de 2020, de https://revistas.uexternado.edu.co/ index.php/contad/article/view/4672

JuanCaballero,A.Z.(2018). RepositorioUniversidad Cooperativa de Colombia. Recuperado el 14 de noviembre de 2020, de https://repository.ucc. edu.co/bitstream/20.500.12494/8461/1/2017 auditoria_forense_contador.pdf

Juriscol, S. (2012). SUIN Juriscol. Recuperado el 13 de noviembre de 2020, de http:// www.suin-juriscol.gov.co/viewDocument. asp?ruta $=$ Decretos $/ 1475504$

Lozano, L. C. (2014). Repositorio Universidad Militar. Recuperado el 12 de noviembre de 2020, de https://repository.unimilitar.edu.co/ bitstream/handle/10654/13537/Importancia $\% 20$ de $\% 201$ as\%20Auditorias.pdf;sequence $=1$

Luna, O. F. (2011). Sistema de Control Interno para Organizaciones. Instituto de investigación en accountability y control IICO. Recuperado el 12 de noviembre de 2020, de https://books.google. es/books?hl=es\&lr=\&id=plsiU8xoQ9EC\&oi=f 


\section{0}

nd\&pg $=$ PP1\&dq $=$ mejoras + en + el + control + inte rno\& ots $=1 \mathrm{NmAKJ} 8301 \& \operatorname{sig}=\mathrm{M} 1 \mathrm{jOO}$ sbrT-5Go aEhXKIv3V0gGr8\#v=onepage\&q\&f=false

Magro, D. A. (2004). Universidad del Salvador. Recuperadoel12denoviembrede2020,dehttps:// racimo.usal.edu.ar/2701/1/P\%C3\%A1ginas desdeTESIS___uni\%270062392___Fraude_ contable_y_sus_efectos_en_el_mercado.pdf

Mantilla, S. A. (2013). Auditoría del control Interno (3 ed.). Ecoe Ediciones. Recuperado el 12 de novimebre de 2020, de https://books.google. es/s?hl=es\&lr=\&id=rMS4DQAAQBAJ\&oi=fn $\mathrm{d} \& \mathrm{pg}=\mathrm{PT} 2 \& \mathrm{dq}=$ auditoria\&ots $=$ PiJbL5voox $\&$

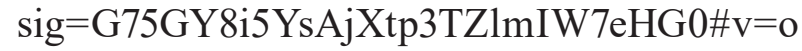
nepage $\& \mathrm{q}=$ auditoria $\& \mathrm{f}=$ false

Martinez, A. A. (2015). Repositorio Unimilitar. Recuperado el 12 de noviembre de 2020, de https://repository.unimilitar.edu.co/ bitstream/handle/10654/7013/EL\%20 F R AUDE $\% 20$ C O R P O R A T I V O \% 20 E N \% 20 L A \% 20 E M P E S A 20 PRIVA DA \% 20 E N \% 20 C O L O M B I A. F0807F089C1A36966A7AA51F?sequence=1

Moncayo, C. (21 de octubre de 2016). Instituto Nacional de Contadores Públicos de Colombia. Recuperado el 14 de noviembre de 2020, de https://www.incp.org.co/el-abuso-de-losrecursos-empresariales-tambien-es-fraude/

Moncayo, C. (10 de julio de 2019). Instituto Nacional de Contadores Públicos. Recuperado el 13 de noviembre de 2020, de https://www.incp.org. co/acuerdo-las-nia-estas-responsabilidadesinformacion-financiera-del-auditor-prevenirfraude/

Monge, E. C. (2010). Las estrategias competitivas y su importancia en la buena gestión de las empresas. Revista Ciencias Económicas, 247276. Recuperado el 14 de noviembre de 2020, de https://revistas.ucr.ac.cr/index.php/economicas/ article/download/7073/6758/0

Mora, J. L. (2006). Biblioteca Universidad San Buenaventura. Recuperado el 12 de noviembre de 2020, de http://biblioteca.usbbog.edu. co:8080/Biblioteca/BDigital/37933.pdf

OCCP. (10 de febrero de 2016). Observatorio Colombiano de Contratación Pública. Recuperado el 13 de noviembre de 2020, de http://occp.co/sites/all/files/Ley\%201778\%20 del\%202016.pdf

Ochoa, M. P. (2016). Enciclopedia Virtual EUMED. Revista Contribuciones a la Economía. Recuperado el 12 de noviembre de 2020, de https://www.eumed.net/ce/2016/3/auditoria. html

Piracón, E. R. (2020). RepositorioUniversidad Santo Tomás. Recuperado el 12 de noviembre de 2020, de https://repository.usta.edu.co/bitstream/ handle/11634/28799/2020alexpiracon. pdf?sequence $=5 \&$ is Allowed $=y$

Portafolio. (15 de julio de 2011). Portafolio. Recuperado el 14 de noviembre de 2020, de https://www.portafolio.co/economia/ finanzas/fraude-devolucion-iva-dian-sumabillon-118916

Pública, F. (2015). Función Pública. Recuperado el 13 de noviembre de 2020, de https://www. funcionpublica.gov.co/eva/gestornormativo/ norma.php? $\mathrm{i}=76745$

Pública, G. N. (2010). Función Pública. Recuperado el 13 de noviembre de 2020, de https://www. funcionpublica.gov.co/eva/gestornormativo/ norma.php? $\mathrm{i}=40032$

Ramírez, M. (2013). Scielo. Recuperado el 13 de noviembre de 2020, de http://www.scielo.org. co/pdf/cuadm/v29n50/v29n50a08.pdf 
República, P. d. (1990). Ministerio de Educación. Recuperado el 12 de noviembre de 2020, de https://www.mineducacion.gov.co/1621/ articles-104547_archivo_pdf.pdf

República, P. d. (12 de julio de 2020). Presidencia de la República. Recuperado el 13 de noviembre de 2020, de http://wp.presidencia.gov.co/sitios/ normativa/leyes/Documents/Juridica/Ley\%20 1474\%20de\%2012\%20de\%20Julio\%20de\%20 2011.pdf

Rodriguez, L. M. (05 de noviembre de 2017). Gerencie. Recuperado el 13 de noviembre de 2020, de https://www.gerencie.com/auditoriaforense.html

Rodríguez, M. A. (26 de julio de 2016). Auditool. Recuperado el 12 de noviembre de 2020, de https://www.auditool.org/blog/controlinterno/4426-por-que-fallan-los-sistemas-decontrol-interno-de-las-empresas

Saldarriaga, A. L. (2011). La Auditoría Forense: Un campo en Potencia. Adversia: Revista virtual de Estudiantes de Contaduría Pública. Recuperado el 12 de noviembre de 2020, de https://revistas.udea.edu.co/index.php/adversia/ article/download/10956/10049/

Santamaría, F. (29 de enero de 2020). La importancia de entender la lógica de los inversionistas. Red Forbes Colombia. Recuperado el 14 de noviembre de 2020, de https://forbes. co/2020/01/29/red-forbes/la-importancia-deentender-la-logica-de-los-inversionistas/

Semana. (13 de junio de 2020). Semana. Recuperado el 14 de noviembre de 2020, de https://www. semana.com/nacion/articulo/diez-anos-delescandalo-del-carrusel-de-la-contratacion-enbogota/679516/

Semana, R. (24 de enero de 2017). Revista Semana. Recuperado el 13 de noviembre de
2020, de https://www.semana.com/odebrechtescandalo-de-corrupcion/513209/

Senado, S. d. (5 de noviembre de 2020). Secretaría del Senado. Recuperado el 12 de noviembre de 2020, de http://www.secretariasenado. gov.co/senado/basedoc/ley_1314_2009. html\#: :text $=$ Por $\% 201 \mathrm{a} \% 20$ cual $\% 20 \mathrm{se} \% 20$ regulan,responsables $\% 20 \mathrm{de} \% 20$ vigilar $\% 20$ su\%20cumplimiento.

Suárez, R. G. (2019). Auditoría y revisoría fiscal (1 ed.). Bogotá: Ecoe Ediciones. Recuperado el 11 de noviembre de 2020, de http://www. ebooks 7-24.com.bdbiblioteca.ufps.edu. co:2048/?il $=9310$

Sura, G. (23 de febrero de 2018). Grupo Sura. Recuperado el 13 de noviembre de 2020, de https://sura-am.com/sites/default/files/ inline-files/sura-asset-management-politicaprevencion-fraude-corrupcion.pdf

Triana, N. C. (2014). Repositorio Universidad Militar Nueva Granada. Recuperado el 14 de noviembre de 2020, de https://repository. unimilitar.edu.co/handle/10654/13185

UMNG, F. (2020). FAEDIS UMNG. Recuperado el 13 de noviembre de 2020, de http://virtual.umng. edu.co/distancia/ecosistema/ovas/contaduria_ publica/auditoria_forense/unidad_6/DM.pdf

Vaca, R. M. (15 de octubre de 2020). Actualicese. Recuperado el 12 de noviembre de 2020, de https://actualicese.com/auditoria-forensecuales-son-sus-caracteristicas-y-que-camposde-aplicacion-tiene/\#

Valle, O. (2012). Organización de los Estados Iberoamericanos. Recuperadoel 13 denoviembre de 2020, de https://www.oei.es/historico/idie/ mONITOREOEINDICADORES.pdf 